\title{
AN ESCHERICHIA COLI CONCENTRATOR USING MAGNETIC PARTICLES IN A MICROFLUIDIC CHANNEL FOR THE URINARY TRACT INFECTION (UTI) APPLICATION
}

\author{
Yongmo Yang, Sangpyeong Kim, and Junseok Chae \\ School of Electrical, Computer and Energy Engineering (ECEE), Arizona State University \\ Tempe, Arizona 85287-5706, United States of America
}

\begin{abstract}
This paper reports a miniaturized lab-on-a-chip device to detect Escherichia coli (E. coli) from simulated urine using magnetic particles (MPs) in a microfluidic channel. The lab-on-achip device consists of two chambers connected in series, E. coli concentration and sensing chamber, integrated with a label free impedance sensor to detect the concentration of E. coli for urinary tract infection (UTI) application. The two chamber configuration is designed to reduce potential false-positive response caused by proteins in urine. The integrated impedance sensor in the concentration chamber shows little impedance change in different E. coli concentrations due to masking effect by proteins while the one in the sensing chamber shows a significant $(30 \mathrm{k} \Omega$ ) change between control (no E. coli) and a concentration of $6.4 \times 10^{4}$ $\mathrm{CFU} / \mathrm{mL}$, close to the threshold of UTI infection, which is above $10^{5} \mathrm{CFU} / \mathrm{mL}$. This demonstrates the effectiveness of the dualchamber design to detect $E$. coli for the UTI application.
\end{abstract}

\section{INTRODUCTION}

Urinary Tract Infection (UTI) is one of the common infections in human, resulting in 8 million hospital visits in US annually [1]. The urine of UTI patients may contain a microbial strain, which can cause UTI, such as Escherichia coli, Proteus mirabilis, Klebsiella, Enterobacter, Enterococcus, Staphylococcus saprophyticus and others [2]. Among them, E. coli is responsible for up to $80 \%$ of UTI [2]. Current standard methods of E. coli detection include multiple-tube fermentation, membrane filter and plate count [3]. Among them, the plate count method is the most widely used, which cultures $E$. coli on an agar plate and counts cultured colony forming units (CFU). If CFU exceeds $10^{5}$ $\mathrm{CFU} / \mathrm{mL}$, the patient has a high chance of having symptomatic UTI [4]. Typically, people do not have symptoms below $10^{5}$ $\mathrm{CFU} / \mathrm{mL}$ [5]. The plate count method offers accurate diagnose, however it is not a feasible method when patients need an immediate medical treatment because it requires lengthy, from 24 to $48 \mathrm{hrs}$, cultivation of urine samples and laborious procedure. In order to avoid such lengthy processes, alternative methods have been developed. Goodridge et al. used a fluorescent bacteriophage assay (FBA), Subramanian et al. developed immunosensor techniques to utilize antibody-derivatized surface plasmon resonance devices, and Wang et al. used piezoelectric crystals and enzyme-linked immunosorbent assays (ELISA) techniques [6-8]. Other researchers developed electrochemical impedance spectroscopy (EIS) to detect E. coli by monitoring the binding of antibody-antigen reaction [9-13]. All those methods offer a quick diagnose of $E$. coli; however capturing efficiency of $E$. coli is low.

In this paper, we take advantage of microfluidics in immunomagnetic separation (IMS) to overcome the limitation, low capturing efficiency. IMS uses magnetic particles (MPs) that are covered by anti-E. coli antibody to capture $E$. coli. The captured $E$. coli is transported via microfluidic channels and the impedance of $E$. coli-immobilized magnetic beads is measured to estimate the concentration of $E$. coli. The impedance results are verified by counting cultured colonies of $E$. coli, the current standard practice.

\section{DESIGN}

The schematic of the lab-on-a-chip device is illustrated in Figure 1. The E. coli concentrator/detector device consists of two mechanical valves [14] and two chambers, concentration and sensing, to reduce non-specific absorption by proteins potentially co-existing with $E$. coli in urine.

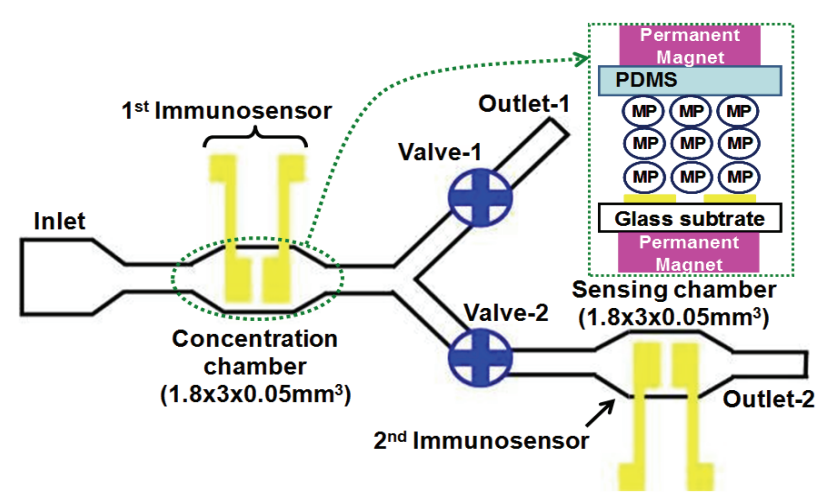

Figure 1: A schematic of the lab-on-a-chip E. coli concentrator/detector. Two separate (concentration and sensing) chambers capture and sense E. coli, respectively. Magnetic particles (MPs) inside the chambers are manipulated by external magnets.

Two mechanical valves control the fluidic stream to Outlet 1 and the sensing chamber. Two immunosensors to measure impedance are integrated in the concentration and sensing chambers and made of two gold $(\mathrm{Au})$ electrodes. MPs were retained in the chamber by placing, two permanent magnets above and beneath of the chambers. Figure 2 shows the length of the chambers as a function of $E$. coli capturing rate.

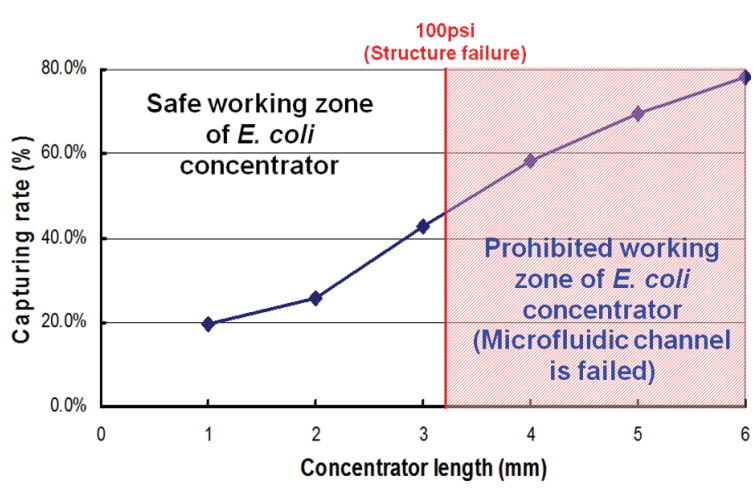

Figure 2: Design criteria for concentration and sensing chamber: the length of the concentrator chamber is determined by the structural failure limit, 100 psi. 
As the length of the chamber increases the capturing rate enhances. Practical limitation exists; as the length increases the fluidic pressure increases as well. The current structure of the labon-a-chip device allows up to 100 psi where the structural failure starts. Thus, we chose the length of the chambers as $3 \mathrm{~mm}$.

\section{Fabrication}

Similar to our previous approaches, we used a replica molding technique using a silicon mold [15]. This two-masks process is shown in Figure 3.

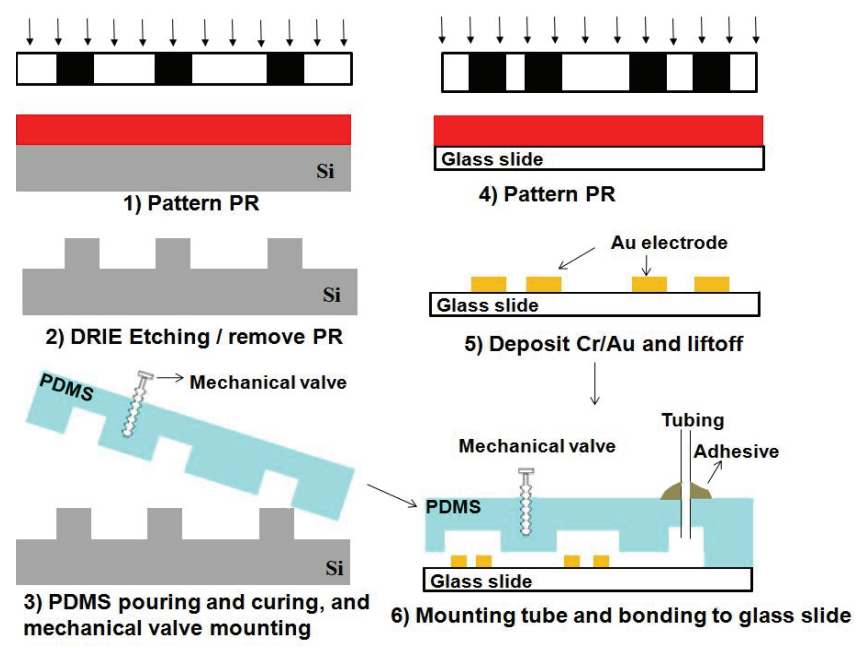

Figure 3: Fabrication flow: forming the top using the replica molding technique (1-3) and the bottom substrate that has electrodes for impedance measurements (4-6).

The silicon mold fabrication starts from photoresist (PR) coating and patterning for the concentrator, followed by Deep RIE etching, $\sim 10 \mu \mathrm{m}$. PDMS (Polydimethylsiloxane) was mixed (10:1) with base and curing agents and then poured on the silicon mold. After 20 min curing in an oven at $80{ }^{\circ} \mathrm{C}$, the PDMS replicas were peeled off, and two mechanical valves were assembled on the PDMS replica [14]. Then, PR was coated on the glass slide substrate and lithographically patterned. $\mathrm{Cr} / \mathrm{Au}$ films for electrodes were evaporated (Edward II). The surfaces of the glass slide and PDMS replica were treated by oxygen plasma (Harrick Plasma) for $1 \mathrm{~min}$ at $100 \mathrm{~W}$. Finally the PDMS top was bonded to the glass slide, and they were cured in the oven at $80{ }^{\circ} \mathrm{C}$ for 40 min. Figure 4 shows the fabricated lab-on-a-chip device.

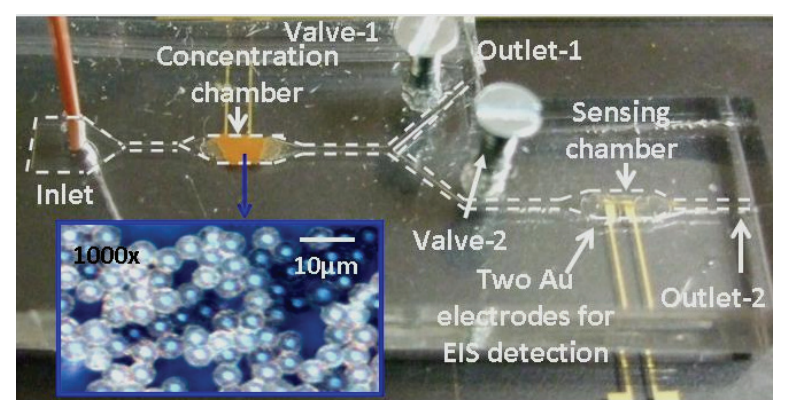

Figure 4: Fabricated lab-on-a-chip E. coli concentrator/sensor (permanent magnets are not shown).

\section{EXPERIMENTAL \\ Reagent}

PDMS (Sylgard 184 elastomer kit) was obtained from Dow Corning. MPs from Spherotech, Inc were $4.5 \mu \mathrm{m}$ in diameter and coated with $\mathrm{COOH}$-terminated self-assembled monolayers (SAM) on the surface. Sodium acetate (NaAc), 1-ehyl-3-(3dimethylaminopropyl) carbodiimide (EDC) and $\mathrm{N}-$ hydrosuccinimide (NHS) were obtained from Pierce and GE health. Phosphate buffered saline solution (PBS) from Fisher scientific was used as a washing solution. Simulated urine was a cocktail of $\mathrm{CaCl}_{2}-\mathrm{H}_{2} \mathrm{O}, \mathrm{Na}_{2} \mathrm{SO}_{4}, \mathrm{MgSO}_{4}-7 \mathrm{H}_{2} \mathrm{O}, \mathrm{NH}_{4} \mathrm{Cl}, \mathrm{KCl}$, Urea, Creatinine and tryptic soy. E. coli $K-12$ (ATCC 10798) was obtained from American Type Culture Collection. The pure culture of $E$. coli $K-12$ was prepared in a tryptic soy broth at $37^{\circ} \mathrm{C}$.

\section{Test setup}

MPs were immobilized with anti-E. coli antibody. MPs coated with $\mathrm{COOH}-\mathrm{SAM}$ were mixed with EDC and NHS in order to activate the functional group of SAM, followed by PBS wash. The anti-E. coli antibody was blended with $10 \mathrm{mM} \mathrm{NaAc}$, and the mixture of them was mixed with MPs to conjugate the anti-E. coli antibody with activated $\mathrm{COOH}$-terminated SAM on the surface of MPs. In order to avoid non-specific absorption, Bovine serum albumin (BSA) was added with MPs. We prepared different concentrations of $E$. coli solution $(100 \%, 10 \%, 1 \%, 0.1 \%$, and $0.01 \%$ ) with BSA in simulated urine.

Figure 5 shows the characterization setup. A syringe pump delivered MPs, mannitol solution and a mixture of E. coli, albumin and simulated urine to the concentration chamber. Permanent magnets were used for manipulating MPs. MPs, mannitol solution, and the sample were flowed by $5 \mu \mathrm{L} / \mathrm{min}, 2 \mu \mathrm{L} / \mathrm{min}$ and $2 \mu \mathrm{L} / \mathrm{min}$, relatively. A LCR meter measured impedance at concentration and sensing chambers and the data were recorded via LabVIEW.

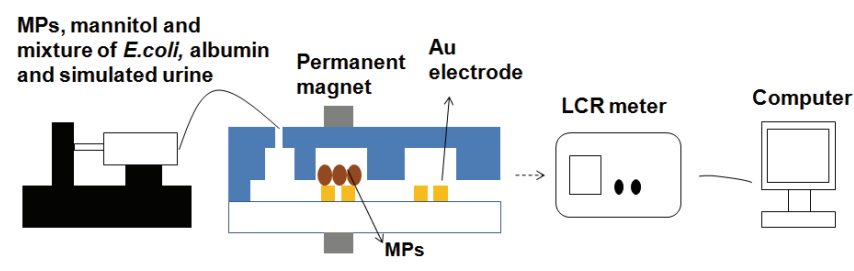

Figure 5: Characterization setup of E. coli lab-on-a-chip device.

\section{Test Procedure}

MPs conjugated with anti-E. coli antibody were injected inside the concentration chamber. Two permanent magnets were placed above and beneath of the chambers to control MPs release and retention. When valve- $1 / 2$ were open/close, we retained MPs in the concentration chamber and flew $10 \mu \mathrm{L}$ of $K-12 \mathrm{E}$. coli and albumin $(1 \mathrm{mg} / \mathrm{mL})$ in simulated urine as shown in Figure 6(a). After $E$. coli was captured by anti-E. coli antibody on the MPs, we flew $90 \mu \mathrm{L}$ of $0.1 \mathrm{M}$ mannitol solution and the impedance sensor in the concentration chamber measured the impedance. When valve- $1 / 2$ were close/open and the two permanent magnets guided the MPs conjugated with $E$. coli to the sensing chamber, the impedance of the MPs was measured again as shown in Figure 6(b). The mannitol solution was used to allow E. coli alive at high solution impedance. 

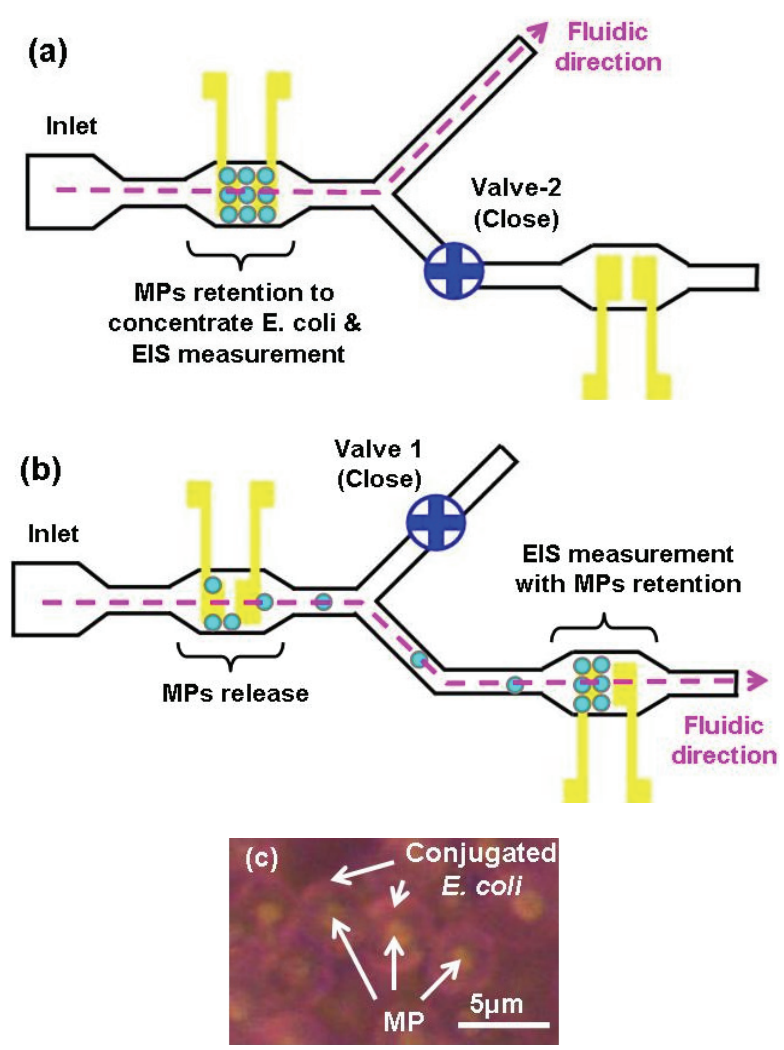

Figure 6: E. coli concentration and impedance measurements procedure: a) MPs retention, E. coli insertion, and measurement in the concentration chamber, b) MPs release, retention, and measurement in the sensing chamber, and c) E. coli conjugated by MPs (after gram stain).

\section{RESULT}

Figure 7 shows the immunosensor measurement in the concentration chamber. In the range of $6.4 \times 10^{4}$ to $6.4 \times 10^{6}$ $\mathrm{CFU} / \mathrm{mL}$, covering the threshold of UTI $\left(10^{5} \mathrm{CFU} / \mathrm{mL}\right)$, the measured impedance changes were almost negligible. This suggests that albumin in the simulated urine was absorbed on the surface of $\mathrm{Au}$ electrodes by the hydrophobic interaction to mask the impedance changes [16-21]. Due to the masking, the impedance measurements did not reflect the change of the $E$. coli concentrations.

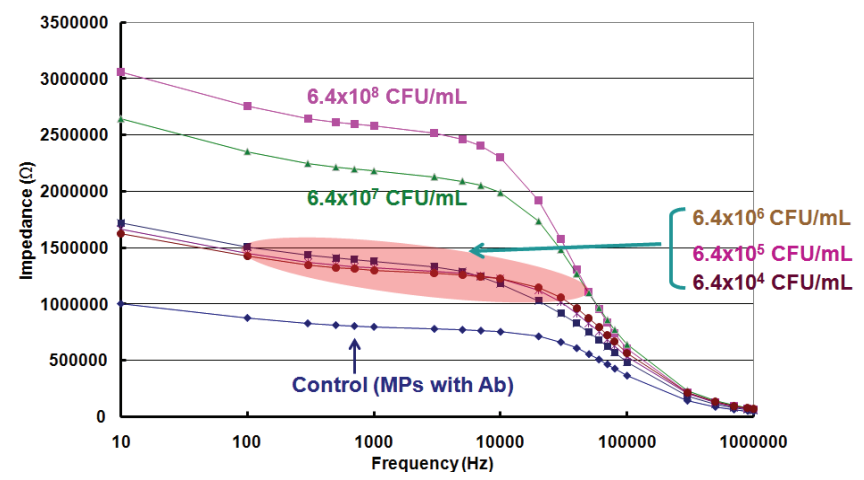

Figure 7: Measured impedance data in the concentration chamber.

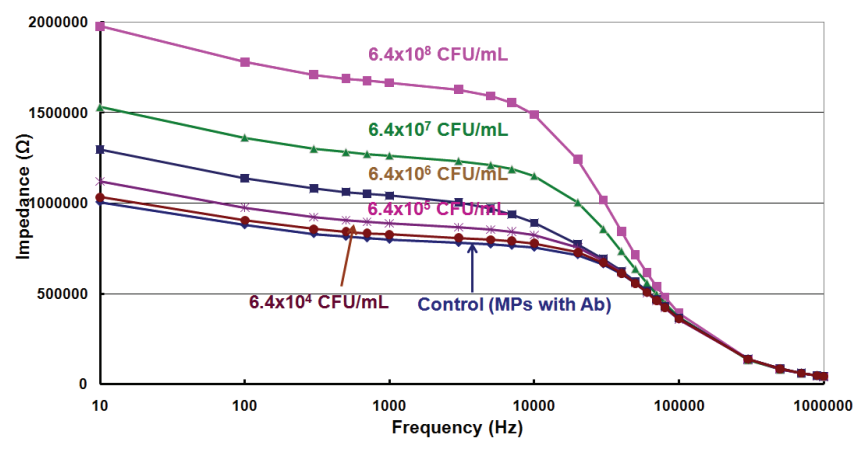

Figure 8: Measured impedance data in the sensing chamber.

Figure 8 shows the impedance measurement in the sensing chamber. As $E$. coli concentration increases, the impedance increases because the membrane of $E$. coli behaves as an insulator [3]. Comparing with the results in the concentration chamber, the impedance changes were bigger in the range from $6.4 \times 10^{4}$ to $6.4 \times 10^{6} \mathrm{CFU} / \mathrm{mL}$. This is because albumin in the sample was removed effectively in the concentration chamber by hydrophobic interaction of albumin with $\mathrm{Au}$ electrodes. This result demonstrates the need of the two chamber configuration to detect E. coli. Figure 9 compares the impedance changes and traditional cultured colony forming unit method, the current standard practice, as a function of the concentration of $E$. coli. The impedance change at $1 \mathrm{kHz}$ was $30 \mathrm{k} \Omega$ between control (no E. coli) and $6.4 \times 10^{4} \mathrm{CFU} / \mathrm{mL}$, which is large enough to distinguish using a commercially-available portable impedance meter [22]. As the concentration of $E$. coli increases the impedance consistently increases. The threshold of UTI, $10^{5} \mathrm{CFU} / \mathrm{mL}$, was shown as a reference in the figure. The impedance data was further verified by counting cultured colonies of $E$. coli in Figure 9(b). We believe that the dual-chamber configuration allows reducing false positive UTI detection in the urine.

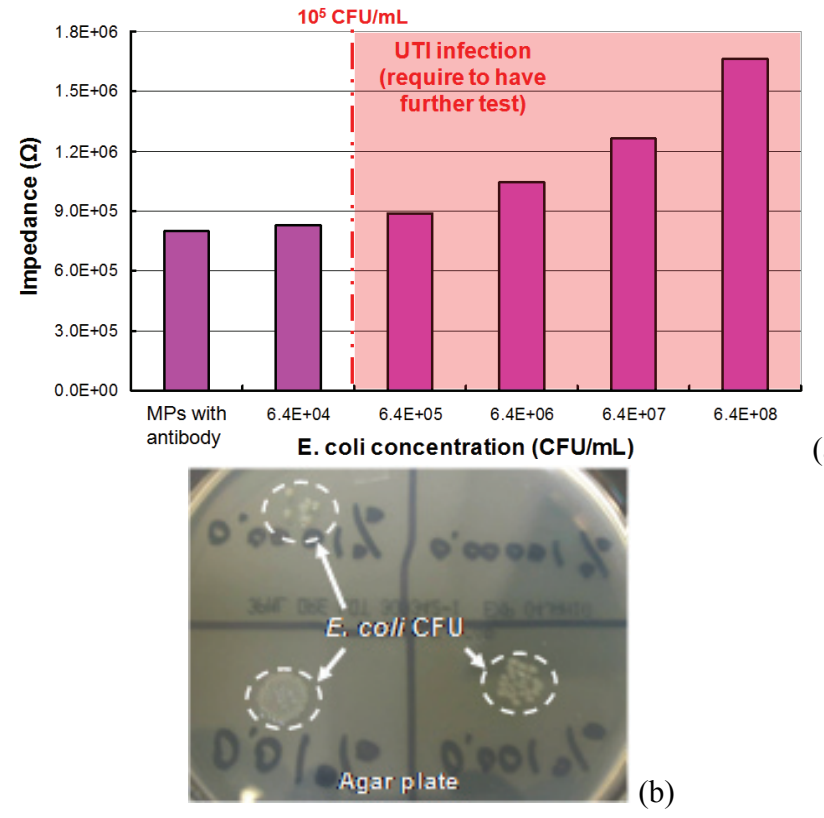

(a)

Figure 9: a) Impedance data at $1 \mathrm{kHz}$, b) E. coli $\mathrm{CFU}$ on a culturing agar plate at the different $E$. coli concentrations. 


\section{CONCLUSION}

We demonstrated a dual-chamber lab-on-a-chip device to detect $E$. coli in simulated urine for UTI applications. The dual chamber configuration allows detecting the concentration of $E$. coli at the presence of protein in the sample. Impedance data at the concentration chamber results show that impedance changes were very little between $6.4 \times 10^{4}$ and $6.4 \times 10^{6} \mathrm{CFU} / \mathrm{mL}$ due to hydrophobic interaction of albumin on $\mathrm{Au}$ electrodes. Albumin masks the electrodes surfaces so that the measured impedance cannot be responsive to the concentration of E. coli in the sample. On the other hand, the impedance data in the sensing chamber did not show such masking effect because albumin was removed effectively in the concentration chamber. The impedance change between the control (no E. coli) and $6.4 \times 10^{4} \mathrm{CFU} / \mathrm{mL}$ was approximately $30 \mathrm{k} \Omega$. Our successful detection result suggests that the dual chamber lab-on-a-chip device may be useful for diagnosis of symptomatic UTI.

Future work includes a reproducibility testing of the $E$. coli lab-on-a-chip device and improving sensitivity of impedance measurement by changing the design of the electrodes for distinguishing asymptomatic and symptomatic UTI.

\section{ACKNOWLEDGEMENT}

The authors thank to Mr. Seokheun Choi for numerous and valuable discussions, and staff in CSSER (Center for Solid-State Electronics Research) at Arizona State University. This work is partially supported by NSF (ECCS-0901440).

\section{REFERENCES}

[1] J. W. Warren and H. L. Mobley, "Urinary Tract Infections", Washington, DC, ASM Press, 1996.

[2] C. M. Kunin, "Detection, prevention and management of urinary tract infections," $4^{\text {th }}$ ed. PA: Lea\&Febiger, 1987.

[3] P. Geng, X. Zhang, W. Meng, Q. Wang, W. Zhang, L. Jin, Z. Feng and $\mathrm{Z}$. Wu, "Self-assembled monolayers-based immunosensor for detection of Escherichia coli using electrochemical impedance spectroscopy", Electrochimica Acta, 53, 14, (2008).

[4] M. T. N. Campanha, S.Hoshino-Shimizu, and M. B. Martinez, "Urinary tract infection: detection of Escherichia coli antigens in human urine with an ELIEDA immunoenzymatic assay", Journal of Public Health, 6, 2 (1999)

[5] C. M. Kunin, L. V. White and T. H. Hua, "A Reassessment of the Importance of "Low-Count" Bacteriuria in Young Women with Acute Urinary Symptoms", Annals of Internal Medicine, 119, 6 (1993)

[6] L. Goodridge, J. Chen and M. Griffiths, "Development and characterization of a fluorescent-bacteriophage assa for detection of Escherichia coli O157:H7", Applied and Environmental Microbiology, 65, 4 (1999).

[7] A. Subramanian, J. Irudayaraj, and T. Ryan, "A mixed selfassembled monolayer-based surface plasmon immunosensor for detection of E. Coli O157:H7”, Biosensors and Bioelectronics, 21, 7 (2006).

[8] N. Wang, M. He and H. C. Shi, "Novel indirect enzymelinked immunosorbent assay (ELISA) method to detect Total E. Coli in water environment", Analytica Chimica Acta, 590, 2 (2007).

[9] C. Ruan, L. Yang and Y. Li, "Immunobiosensor Chips for Detection of Escherichia coli O157:H7 Using Electrochemical Impedance Spectroscopy", Analytical Chemistry, 74, 18 (2002).

[10] V. E. Gomez, S. Campuzano, M. Pedrero and J. M. Pingarron, "Gold screen-printed-based impedimetric immunobiosensors for direct and sensitive Escherichia coli quantisation", Biosensors and Bioelectronics, 24, 11 (2009).

[11] L. Wang, Q. Liu, Z. Hu, Y. Zhang, C. Wu, M. Yang, and P. Wang, "A novel electrochemical biosensor based on dynamic polymerase-extending hybridization for E. Coli O157:H7 DNA detection", Talanta, 78, 3 (2009).

[12] R. Maalouf, C. F. Wirth, J. Coste, H. Chebib, Y. Saikali, O. Vittori, A. Errachid, J. P. Cloarec, C. Martelet and N. J. Renault, "Label-Free Detection of Bacteria by Electrochemical Impedance Spectroscopy: Comparison to Surface Plasmon Resonance", Analytical Chemistry, 79, 13 (2007).

[13] L. Yang and R. Bashir, "Electrical/electrochemical impedance for rapid detection of foodborne pathogenic bacteria", Biotechnology Advances, 26, 2 (2008).

[14] D. B. Weibel, et al., "Torque-Actuated Valves for Microfluidics," Analytical Chemistry, 77 (2005).

[15] Y. Yang and J. Chae, "A miniaturized protein separation using a liquid chromatography column on a flexible substrate," Journal of Micromechanics and Microengineering, 18, 12 (2008).

[16] T. Ederth, P. Claesson and B. Liedberg, "Self-Assembled Monolayers of Alkanethiolates on Thin Gold Films as Substrates for Surface Force Measurements. Long-Range Hydrophobic Interactions and Electrostatic Double-Layer Interactions", Langmuir, 14 (1998)

[17] B. Catimel, A. M. Scott, F. T. Lee, N. Hanai, G. Ritter, S. Welt, L. J. Old, A. W. Burgess, and E. C. Nice, "Direct immobilization of gangliosides onto goldcarboxymethyldextran sensor surfaces by hydrophobic interaction: applications to antibody characterization", Gloycobiology, 8 (1998)

[18] E. Ostuni, L. Yan and G. M. Whitesides, "The interaction of proteins and cells self-assembled monolayers of alkanethiolates on gold and silver", Colloids and Surfaces B: Biointerfaces, 15, 1 (1999)

[19] W. Melander and C. Horvath, "Salt effects on hydrophobic interactions in precipitation and chromatography of proteins: An interpretation of the lyotropic series", Archives of Biochemistry and Biophysics, 183, 1 (1977)

[20] S. Zhang and Y. Sun, "Further studies on the contribution of electrostatic and hydrophobic interactions to protein adsorption on dye-ligand adsorbents", Biotechnology and Bioengineering, 75, 6 (2001)

[21] P. Roach, D. Farrar and C. C. Perry, "Interpretation of Protein Adsorption: Surface-Induced Conformational Changes", Journal of American Chemical Society, 127 (2005)

[22] TOA Electronics, Inc, http://www.toaelectronics.com/spec_sheets/zm-104.pdf

\section{CONTECT}

Y. Yang, tel: +1-602-741-0193; yongmo.yang@asu.edu 